\title{
ANALYTIC FAMILIES OF OPERATORS ON SOME QUASI-BANACH SPACES
}

\author{
MICHAEL CWIKEL AND YORAM SAGHER
}

(Communicated by John B. Conway)

\begin{abstract}
An interpolation theorem for analytic families of operators on some quasi-Banach spaces is proved. The result is applicable to spaces whose quasi-norm is defined by means of a maximal function, for example the various $H_{p}$ spaces on locally compact groups.
\end{abstract}

Introduction. Analytic families of operators serve as an important tool in harmonic analysis. In the framework of Banach spaces they can be defined relatively easily using duality. In the context of quasi-Banach spaces this approach is not available. The importance of some quasi-Banach spaces, for example $H_{p}$ spaces, justifies the development of a corresponding theory even though certain obstacles, such as the absence of a maximum principle for quasi-Banach space valued functions, seem to preclude one from achieving the elegance of the theory in the Banach case. (Note, however, that the apparent simplicity of the Banach space case can be misleading; see e.g. [3].)

The case in which $T_{z} a$ takes values in a finite-dimensional subspace of the range for each $a$ in a dense subset of $A_{0} \cap A_{1}$ has been treated in [9]. The results of [9] are adequate for handling, for example, multipliers on compact groups. In the present paper we trade the finite-dimensionality condition for various other conditions on the spaces and operators and offer a theorem which can conveniently handle multipliers on noncompact groups. Unlike [9] our results here generalize the main result of $[8]$.

For a short exposition of complex interpolation of quasi-Banach spaces (or rather, complex interpolation of their quasi-norms) we refer to [4 and $\mathbf{9}]$.

The Interpolation Theorem. Denote the strip $\{z \in \mathbf{C} \mid 0<$ re $z<1\}$ by $S$. The Poisson kernel for $S$ will be denoted by $d P(\varsigma, z)$, with $\varsigma \in \partial S$ and $z \in S$. Let $\left(B_{0}, B_{1}\right)$ be an interpolation couple of quasi-Banach spaces. Let $B$ be a linear space which is contained in $B_{0}+B_{1}$. We assume that the elements of $B$ are measurable functions on some measure space $\Omega$. We introduce a space $\mathcal{H}(B)$ of $B$-valued functions $h(\varsigma)=h(\varsigma, \omega)$ defined for almost all $\varsigma \in \partial S$. We assume that $H(B)$ is closed under pointwise multiplication by functions $\psi(\varsigma)$ which are boundary values of $H^{\infty}(S)$ functions. The elements of $H(B)$ must also satisfy

$$
\int_{\partial S}|h(\varsigma, \omega)| d P(\varsigma, z)<\infty \quad \text { for a.e. } \omega \in \Omega
$$

Received by the editors December 29, 1986.

1980 Mathematics Subject Classification (1985 Revision). Primary 46M35; Secondary 42B30. 
and

$$
\int_{\partial S} h(\varsigma, \omega) d P(\varsigma, z) \in B \quad \text { for each } z \in S
$$

We assume that there exists an operator $M: B \rightarrow \mathcal{M}^{+}(X, \Sigma, \mu)$ (positive measurable functions on $(X, \Sigma, \mu))$ which satisfies:

(1a) $M h(\varsigma, \cdot)(x)$ is a measurable function of $(\varsigma, x)$ for each $h \in \mathcal{H}(B)$.

(1b) For every $b \in B$ and every $x \in X$ for which $M b(x)$ is defined, $M(\lambda b)(x)$ is also defined for each $\lambda \in \mathbf{C}$ and

$$
M(\lambda b)(x)=|\lambda| M b(x) .
$$

(1c) For each $h \in \mathcal{H}(B)$ there exists a set $E=E(h)$ with $\mu(E)=0$, such that the inequality

$$
M\left(\int_{\partial S} h(\varsigma, \cdot) d P(\varsigma, z)\right)(x) \leq \int_{\partial S} M h(\zeta, \cdot)(x) d P(\varsigma, z)
$$

holds for all $z \in S$ and all $x \notin E$. Furthermore, for all $\psi \in H^{\infty}(S), E(\psi h)=E(h)$.

Let $\left(A_{0}, A_{1}\right)$ be a second interpolation couple of quasi-Banach spaces and let $A$ be a dense linear subspace of $A_{0} \cap A_{1}$. As shown in [9, Theorem 1], the calculation of intermediate quasi-norms for elements of $A$ can be obtained by working entirely within the context of $A$-valued analytic functions.

We assume that for each $z \in \bar{S}$ there is a linear operator $T_{z}: A \rightarrow B$ and that:

(i) For almost every $\omega \in \Omega, T_{z} a(\omega)$ is an analytic function of $z$ on $S$, is continuous in $\bar{S}$ and has admissible growth in $S$, i.e. (cf. $[\mathbf{5}, 10])$

$$
\left\{\begin{array}{l}
\left|T_{s+i t} a(\omega)\right| \leq \exp (A \exp \alpha|t|) \\
\text { for some constants } \alpha=\alpha(a)<\pi \text { and } A=A(a, \omega) .
\end{array}\right.
$$

Furthermore, defining $h_{\varepsilon}(z)=\exp [-\varepsilon \cos (\beta(z-1 / 2))]$, we require that $h_{\varepsilon}(\varsigma) T_{\varsigma} a(\omega)$ $\in \mathcal{H}(B)$ for all choices of $\varepsilon>0$ and $\beta \in(\alpha, \pi)$.

(ii) For each $a \in A, t \in \mathbf{R}$ and $j=0,1$,

$$
\left\|M T_{j+i t} a(\cdot)\right\|_{L_{p_{j}}(X)} \leq K_{j}(t)\|a\|_{A_{j}},
$$

where

$$
K(z)=\exp \left(\int_{-\infty}^{\infty} \log K_{0}(t) d P(i t, z)+\int_{-\infty}^{\infty} \log K_{1}(t) d P(1+i t, z)\right)<\infty .
$$

Given the above hypotheses it follows that for $0<s<1$ and all $a \in A$ :

$$
\left\|M T_{s} a(\cdot)\right\|_{L_{p(s)}(X)} \leq K(s)\|a\|_{\left[A_{0}, A_{1}\right]_{s}},
$$

where $1 / p(s)=(1-s) / p_{0}+s / p_{1}$.

We now turn to the proof of this assertion:

Let $a$ be an element of $\mathcal{A}$. Let $f(z)=\sum_{k} \psi_{k}(z) a_{k}$ where $a_{k} \in A, \psi_{k} \in H^{\infty}(S)$ and $f(s)=a$. (The above sum, like those which follow, is understood to be taken over finitely many terms.) Note also that in view of Proposition 2.5 of [2, p. 210], the condition $\psi_{k} \in H^{\infty}(S)$ (instead of $\psi_{k} \in N^{+}(S)$ ) does not affect the calculation of the value of $\|a\|_{\left[A_{0}, A_{1}\right]_{s}}$. 
Let $\alpha \in(0, \pi)$ and $A=A(\omega)$ be such that (2) is satisfied for all the $a_{k}$ and a.e. $\omega$. Let $\beta \in(\alpha, \pi)$. The function $h_{\varepsilon}(z)$ defined above satisfies $\left|h_{\varepsilon}(z) T_{z} a_{k}(\omega)\right| \leq$ $\exp \left(A e^{\alpha|t|}-(\varepsilon / 2) e^{\beta|t|} \cos (\beta / 2)\right)$, and so, for a.e. $\omega$,

$$
\lim _{|t| \rightarrow \infty}\left|h_{\varepsilon}(s+i t) T_{s+i t} a_{k}(\omega)\right|=0 \quad \text { uniformly in } s .
$$

For each $x \in X$ and $\varsigma \in \partial S$, let

$$
m_{n}(\zeta, x)= \begin{cases}M T_{\zeta} f(\varsigma)(x) & \text { if } M T_{\zeta} f(\varsigma)(x)>1 / n \\ 1 / n & \text { otherwise. }\end{cases}
$$

Let $u_{n}(z, x)=\int_{\partial S} \log m_{n}(\varsigma, x) d P(\varsigma, z)$. For some fixed $x$, if $u_{n}(z, x)$ is finite for one, and therefore all, $z$ in $S$, then we denote its harmonic conjugate in $S$ by $\tilde{u}_{n}$ and define $\varphi_{n}(z, x)=u_{n}(z, x)+i \tilde{u}_{n}(z, x)$. (It will be clear that the estimate (5) below is trivially true if $u_{n}(z, x)=\infty$.) $\left|e^{-\varphi_{n}(z, x)}\right|=e^{-u_{n}(z, x)} \leq n$ and so $e^{-\varphi_{n}(z, x)} \in H^{\infty}(S)$. Furthermore, in view of (4),

$$
e^{-\varphi_{n}(z, x)} h_{\varepsilon}(z) T_{z} f(z)(\omega)=\int_{\partial S} e^{-\varphi_{n}(\varsigma, x)} h_{\varepsilon}(\varsigma) T_{\varsigma} f(\varsigma)(\omega) d P(\varsigma, z)
$$

for a.e. $\omega$. Applying (1c) and (1b) to the last identity yields

$$
\begin{aligned}
& e^{-u_{n}(z, x)}\left|h_{\varepsilon}(z)\right| M\left(T_{z} f(z)\right)(y) \\
& \quad \leq \int_{\partial S} e^{-u_{n}(\zeta, x)}\left|h_{\varepsilon}(\zeta)\right| M\left(T_{\zeta} f(\zeta)\right)(y) d P(\zeta, z)
\end{aligned}
$$

for each $y$ not in the exceptional set for the function $h_{\varepsilon} T_{\varsigma} f(\varsigma)(\omega)$. It follows that this inequality holds with $\varepsilon=1 / k$ for all positive integers $k$ and for all $y$ in the complement of a set $E$ of zero $\mu$-measure, which does not depend on our choice above of $x$. Consequently it is valid for almost all choices of $x$, and in particular for almost all $x \notin E$. Thus, setting $x=y$ and observing that $e^{-u_{n}(\varsigma, x)} M\left(T_{\varsigma} f(\varsigma)\right)(x) \leq 1$, we have that $e^{-u_{n}(z, x)}\left|h_{\varepsilon}(z)\right| M\left(T_{z} f(z)\right)(x) \leq 1$. Letting $\varepsilon=1 / k$ tend to zero we obtain that $M\left(T_{z} f(z)\right)(x) \leq e^{u_{n}(z, x)}$ for a.e. $x$. Now letting $n$ tend to $\infty$, it follows that

$$
\log M\left(T_{z} f(z)\right)(x) \leq \int_{\partial S} \log M\left(T_{\varsigma} f(\varsigma)\right)(x) d P(\varsigma, z)
$$

for almost every $x$. If we set $\partial S_{j}=\{j+i t \mid t \in \mathbf{R}\}$ for $j=0,1$, and choose $z=s$, then (5) can be rewritten as

$$
\begin{aligned}
M\left(T_{s} f(s)\right)(x) \leq & \left(\exp \int_{\partial S_{0}} \log M\left(T_{\varsigma} f(\varsigma)\right)(x) \frac{d P(\varsigma, s)}{1-s}\right)^{1-s} \\
& \times\left(\exp \int_{\partial S_{1}} \log M\left(T_{\varsigma} f(\varsigma)\right)(x) \frac{d P(\varsigma, s)}{s}\right)^{s}
\end{aligned}
$$

and so, since the function $M\left(T_{\varsigma} f(\varsigma)\right)(x)$ is jointly measurable, we can apply Hölder's inequality, followed by a limiting case of Minkowski's inequality (see [8]) to obtain (3).

We continue with some remarks about the preceding result and its proof:

(i) Note that the proof does not use the measure space structure of the set $\Omega$ and thus the theorem holds if $\Omega$ is merely an abstract set, provided "a.e. $\omega$ " is 
replaced by "all $\omega$ ". The two versions are essentially equivalent if $A$ is the span of a countable set of elements. In many applications of which we are aware, the action of $M$ requires $\Omega$ to be a measure space, hence our choice of formulation. On the other hand, in the context of the application to $H_{p}$ spaces (cf. below) one could, instead of taking $\Omega=\mathbf{R}^{n}$, take $\Omega$ to be a space of test functions, and $B$ to be a space of distributions $f$ with $f(\omega)=\langle f, \omega\rangle$ for each test function $\omega$.

(ii) We can also observe that the couple $\left(B_{0}, B_{1}\right)$ plays essentially no role in the statement or the proof of the result. We introduce it merely for conceptual convenience. For example (cf. the application below), if $B_{j}$ is the space of elements $b$ for which $M b \in L_{p_{j}}$ with quasi-norm $\|b\|_{B_{j}}=\|M b\|_{L_{p_{j}}}$, then condition (ii) above on $T_{z}$ is a reformulation of the requirement that $T_{j+i t}$ is a bounded map from (a dense subclass of) $A_{j}$ into $B_{j}$.

(iii) One might expect condition (1c) imposed above on $M$ to imply that $M$ is sublinear, i.e. $M\left(b_{0}+b_{1}\right)(x) \leq M\left(b_{0}\right)(x)+M\left(b_{1}\right)(x)$ for a.e. $x$ and for every $b_{0}$ and $b_{1}$ in $B$, but the following simple example shows that this is not the case. Let $B=\mathbf{C}$ and $\Omega$ be a singleton set and let

$$
M(b)= \begin{cases}0 & \text { if }|b|<1, \\ |b|-1 & \text { otherwise. }\end{cases}
$$

(However if $(\partial S, d P(\cdot, z))$ were to be replaced by a nonatomic measure space with infinite total measure, if it were known that $M(0)=0$, and if $H(B)$ contained, for example, all functions of the form $\chi_{E_{0}}(\varsigma) b_{0}+\chi_{E_{1}}(\varsigma) b_{1}$, then the counterpart of (1c) would imply sublinearity as well as the counterpart of (1b).)

(iv) For a given particular analytic family of operators $\left\{T_{z}\right\}$ the "optimal" way to choose $B$ and $H(B)$ in order to minimize the hypotheses required for (3) to hold, is of course to let $B=\left\{T_{z} a \mid a \in A, z \in \bar{S}\right\}$ and $\mathcal{H}(B)$ be the space of all finite sums of functions of the form $\psi(\varsigma) T_{\varsigma} a(\omega)$ for all choices of $a \in A$ and $\psi \in H^{\infty}(S)$.

(v) In many applications $M$ will be a maximal operator of the form $M b(x)=$ $\sup _{\delta}\left|U_{\delta} b(x)\right|$, where each $U_{\delta}$ is a linear operator from $B$ into $\mathcal{M}(X, \Sigma, \mu)$. The verification of the hypotheses for our theorem can then be rather straightforward. We give an example of this in the next section.

(vi) It is possible with very minor modifications to formulate and prove a variant of our result in which the spaces $L_{p_{0}}$ and $L_{p_{1}}$ are replaced by quasi-Banach lattices $E_{0}$ and $E_{1}$ of measurable functions on $(X, \Sigma, \mu)$ which must have the property that

$$
\left\|\exp \int_{\partial S_{j}} \log |h(\varsigma, \cdot)| d \mu_{j}(\varsigma)\right\|_{E_{j}} \leq 1
$$

whenever $h(\zeta, x)$ is a jointly measurable function such that $\|h(\zeta, \cdot)\|_{E_{j}} \leq 1$ for a.e. $\varsigma \in \partial S_{j}$ and $d \mu_{j}$ is a probability measure on $\partial S_{j}$ (cf. the quasi-Banach lattices studied by Kalton in $[6,7])$. In this case $(6)$ with probability measures $d \mu_{0}=$ $d P(\varsigma, s) /(1-s)$ and $d \mu_{1}=d P(\varsigma, s) / s$ yields immediately that $\left\|M T_{s} a(\cdot)\right\|_{E_{0}^{1-s} E_{1}^{s}} \leq$ $K(s)\|a\|_{\left[A_{0}, A_{1}\right]_{s}}$, where the "Calderón product" lattice $E_{0}^{1-s} E_{1}^{s}$ is defined as in [1]. To show that (7) is satisfied, it is of course sufficient to show that

$$
\left\|\left(\int_{\partial S_{j}}|h(\varsigma, \cdot)|^{r} d \mu_{j}(\varsigma)\right)^{1 / r}\right\|_{E_{j}} \leq 1
$$


for some positive $r$, and this is clearly true, for example, for $L(p, q)$ spaces with suitable quasi-norms. Indeed, if we apply our result to the case of $L(p, q)$ spaces and define $M$ simply by $M f(x)=|f(x)|$, then we obtain the main theorem of [8].

Applications. The above interpolation theorem has natural applications to $H_{p}$ spaces of all types as well as to Sobolev spaces. We show, for example, how its conditions can be verified in the case of $H_{p}\left(\mathbf{R}^{n}\right)$ spaces for $p>0$.

Let $\varphi$ be a $C^{\infty}$ function with compact support, $\varphi_{\delta}(x)=\delta^{-n} \varphi(x / \delta)$, and $M f(x)=\sup _{\delta>0}\left|\varphi_{\delta} * f\right|(x)$. A tempered distribution $f$ belongs to $H_{p}$ if and only if $M f \in L_{p}$. We consider the case where $B_{j}=H_{p_{j}}\left(\mathbf{R}^{n}\right)$ for $j=0,1$. In this case $T_{z} a$ may not be a function on $\bar{S} \times \mathbf{R}^{n}$ but this can be overcome by convolving it with a Schwartz function. The resulting quasi-norm inequalities do not depend on the choice of Schwartz function and thus lead to the full interpolation result. Consequently the assumption in the following theorem, that $T_{z} a$ is always a function, is not a restriction in applications.

THEOREM. Let $\left(A_{0}, A_{1}\right)$ and $\mathcal{A}$ be as above and suppose that, for each $z \in \bar{S}$, the operator $T_{z}: A \rightarrow C^{\infty}\left(\mathbf{R}^{n}\right) \cap\left(H_{p_{0}}+H_{p_{1}}\right)$ satisfies the further properties:

(i) $\left\|T_{j+i t} a\right\|_{H_{p_{j}}} \leq K_{j}(t)\|a\|_{A_{j}}$ for all $a \in \mathcal{A}, t \in \mathbf{R}$, and $j=0,1$.

(ii) For each $a \in A$, the function $T_{z} a(\omega)$ is a measurable function of $(z, \omega)$ on $\bar{S} \times \mathbf{R}^{n}$, which also satisfies (2) for suitable $\alpha(a)$ and $A(a, \omega)$, depends analytically on $z \in S$ for each $\omega \in \mathbf{R}^{n}$ and is continuous up to the boundary at all points $\varsigma \in \partial S$. Furthermore, for each compact subset $F \subset \mathbf{R}^{n}$, each $\varepsilon>0$ and every choice of $\beta \in(\alpha(a), \pi), \int_{F} \int_{\partial S}\left|h_{\varepsilon}(\varsigma) T_{\zeta} a(\omega)\right| d P(\zeta, z) d \omega<\infty$, where $h_{\varepsilon}$ is the function defined following (2).

Then $\left\|T_{s} a\right\|_{H_{p(s)}} \leq K(s)\|a\|_{\left[A_{0}, A_{1}\right]_{s}}$ for each $a \in \mathcal{A}$ and $s \in(0,1)$.

PROOF. We apply the result of the preceding section, taking $B=C^{\infty}\left(\mathbf{R}^{n}\right) \cap$ $\left(H_{p_{0}}+H_{p_{1}}\right)$ and choosing $\mathcal{H}(B)$ to be the space of all functions $h(\xi, \omega)$ which are finite sums of terms of the form $\psi(\varsigma) h_{\varepsilon}(\varsigma) T_{\varsigma} a$, where $a \in \mathcal{A}, \varepsilon>0, \psi \in H^{\infty}(S)$ and the value of $\beta$ for $h_{\varepsilon}$ is chosen in the range $(\alpha(a), \pi)$. Each such $h(\varsigma, \omega)$ is clearly measurable in $\partial S \times \mathbf{R}^{n}$. Let

$$
U_{\delta} g(x)=\delta^{-n} \int_{\mathbf{R}^{n}} \varphi\left(\frac{x-\omega}{\delta}\right) g(\omega) d \omega .
$$

Since $\int_{F} \int_{\partial S}|h(\zeta, \omega)| d P(\varsigma, z) d \omega<\infty$ for each compact $F$ and since $\varphi$ is bounded and has compact support, we can apply Fubini's theorem to obtain

$$
U_{\delta}\left(\int_{\partial S} h(\zeta, \cdot) d P(\varsigma, z)\right)(x)=\int_{\partial S} U_{\delta} h(\zeta, \cdot)(x) d P(\varsigma, z) .
$$

Since the last integrand depends continuously on $\delta$, we have that $M h(\zeta, \cdot)(x)$ is a measurable function of $(\varsigma, x)$ and so

$$
\left|U_{\delta}\left(\int_{\partial S} h(\varsigma, \cdot)(x) d P(\varsigma, z)\right)(x)\right| \leq \int_{\partial S} M h(\varsigma, \cdot)(x) d P(\varsigma, z) .
$$

Taking the supremum over $\delta$ establishes (1c) and indeed in this case $E(h)=\varnothing$ for each $h$. All other required conditions on $\mathcal{H}(B)$ and $T_{z}$ are easily seen to hold and so we obtain (3) and consequently complete the proof of the theorem. 


\section{BIBLIOGRAPHY}

1. A. P. Calderón, Intermediate spaces and interpolation, the complex method, Studia Math. 24 (1964), 113-190.

2. R. R. Coifman, M. Cwikel, R. Rochberg, Y. Sagher and G. Weiss, A theory of complex interpolation for families of Banach spaces, Adv. in Math. 43 (1982), 203-229.

3. M. Cwikel and S. Janson, Interpolation of analytic families of operators, Studia Math. 79 (1984), 61-71.

4. M. Cwikel, M. Milman and Y. Sagher, Complex interpolation of some quasi-Banach spaces, J. Funct. Anal. 65 (1986), 339-347.

5. I. I. Hirschman, A convexity theorem for certain groups of transformations, J. Analyse Math. 2 (1953), 209-218.

6. N. J. Kalton, Convexity theorems for non-locally convex lattices, Glasgow Math. J. 258 (1984), 141-152.

7. __ Plurisubharmonic functions on quasi-Banach spaces, Studia Math. (to appear).

8. Y. Sagher, On analytic families of operators, Israel J. Math. 7 (1969), 350-356.

9. __ Interpolation of some analytic families of operators, Proc. U.S. Swedish Seminar on Function Spaces and Applications, Lecture Notes in Math., vol. 1302, Springer.

10. E. M. Stein, Interpolation of linear operators, Trans. Amer. Math. Soc. 83 (1956), 482-492.

Department of Mathematics, University of Chicago, Chicago, Illinois 60637

Department of Mathematics, University of Illinois, Chicago, Illinois 60680 32000

Current address (Michael Cwikel): Department of Mathematics, Technion I. I. T., Haifa, Israel, 